\title{
Reconstrucción de pared torácica por carcinoma mamario. Presentación de un caso
}

\author{
Chest wall reconstruction due to breast carcinoma. Case report
}

\author{
Adriana Natalia Cotto Perroni', Sergio Martínez² ${ }^{2}$ Rocío Nacir ${ }^{3}$
}

\section{RESUMEN}

El propósito de la presentación de este caso es demostrar nuestra experiencia en el Servicio de Cirugía Plástica y Reconstructiva de resolver casos complejos de defectos de pared torácica con colgajos locales y a distancia y valorizar su uso en reconstrucciones complejas. En los últimos años trabajamos coordinadamente con el Servicio de Mastologia de nuestro hospital y este caso fue resuelto también en conjunto con el Servicio de Cirugía Torácica.

Palabras claves: reconstrucción de pared torácica, carcinoma de mama, colgajo dorsal ancho.

\begin{abstract}
The purpose of the presentation of this case is to demonstrate our experience in the service of plastic and reconstructive surgery to solve complex an distance cases of thoracic wall defects and value their use in complex reconstructions. In recent years we worked in coordination with the hospital's mastology service and this case was also resolved in conjunction with the thoracic surgery service.
\end{abstract}

Key words: wall defects reconstruction, breast carcinoma, wide dorsal flap.

\section{INTRODUCCIÓN}

En Argentina, el cáncer de mama es responsable del mayor número de muertes por tumores malignos entre las mujeres: representa el $21 \%$ del total de muertes por cáncer. En la provincia del Chaco las pacientes afectadas por cáncer de mama son pacientes jóvenes, algunas de ellas apenas superan los 30 años y con estadios avanzados, sobre todo en el interior de la provincia.

El manejo quirúrgico del cáncer de mama continúa evolucionando y sigue siendo un componente clave del tratamiento y la curación ${ }^{4}$.

El tratamiento interdisciplinario es fundamental para estas pacientes. Muchas de ellas consultaban luego de años de la mastectomía o no consultaban en nuestro hospital; actualmente se está realizando la reconstrucción mamaria coordinando con el Servicio de Mastologia y se plantea la reconstrucción de acuerdo a cada

1. Médico de planta del Servicio de Cirugía Plástica, Reconstructiva y Quemados. Hospital Julio C. Perrando. Miembro titular SACPER

2. Jefe de Servicio de Cirugía Plástica Reconstructiva y Quemados del Hospital Julio C. Perrando. Miembro titular SACPER

3. Residente del segundo año del Servicio de Cirugía Plástica y Quemados del Hospital Julio C. Perrando, Servicio de cirugía Plástica, Reconstructiva y Quemados. Resistencia, Chaco

$\square$ Correspondencia: Dra. Adriana Natalia Cotto Perroni. naticotto@ hotmail.com

Los autores no declaran conflictos de intereses

Recibido: 03/09/2019 / Aceptado: 10/09/2019 caso. Uno de los principales beneficios de la reconstrucción inmediata es evitar el trauma psicológico posterior a la amputación mamaria ${ }^{5}$, pero no está indicada en todas las pacientes.

La reconstrucción puede ser efectuada utilizando una variedad de técnicas, que pueden involucrar el uso de tejidos autólogos, implantes o una combinación de ambos. Los factores del paciente (tales como el hábito corporal, comorbilidades y procedimientos quirúrgicos previos) afectan sobre qué técnicas son realmente ofrecidas en cada caso en particular 5 .

Las pacientes que requieren radioterapia posmastectomía pueden ser particularmente vulnerables a las complicaciones posreconstrucción, la radiación aumenta el riesgo de complicaciones, tanto en las pacientes que reciben implantes mamarios como en aquellas sometidas a reconstrucción autóloga.

\section{MATERIALES Y MÉTODO}

Paciente de sexo femenino de 41 años, con antecedentes de adenomastectomía izquierda en 2012 por carcinoma ductal infiltrante indiferenciado mamario estadio III B RE + $\mathrm{RP}+$. En 2013 realizó quimioterapia y radioterapia.

En el mes de octubre de 2018 la paciente presenta dolor en región anterior de tórax. Se le realizó centellograma óseo de cuerpo entero que muestra compromiso de pared torácica a nivel esternal, costal y de partes blandas, y se practicó resección de pared torácica a nivel esternal y de tres arcos costales y reconstrucción con barras estabilizadoras y malla de doble contacto por parte del servicio de cirugía torácica. 


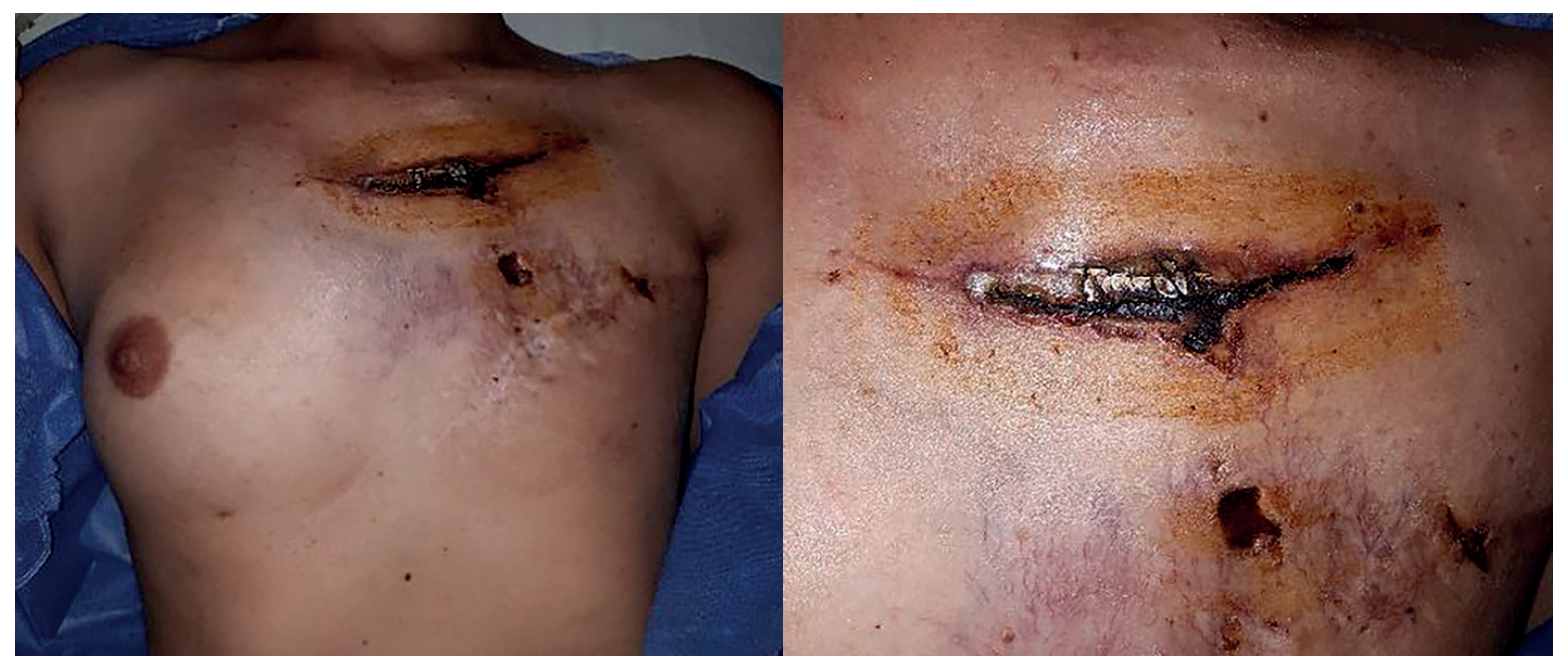

Figuras 1 y 2. Posoperatorio alejado de mastectomía radical más estabilización torácica por carcinoma invasor estadio III B.

Luego de 3 meses del procedimiento, la paciente presentó exposición de material protésico, motivo por el cual el servicio de cirugía torácica realiza la interconsulta al servicio de cirugía plástica (Figuras 1 y 2).

Se plantea la extracción del material y la realización de un colgajo de dorsal ancho para cobertura, se realizaron estudios prequirúrgicos y un estudio eco-Doppler vascular para constatar la permeabilidad de los vasos de la arteria axilar y toracodorsal. El pedículo vascular del colgajo es la arteria toracodorsal, rama de la subescapular, la cual debe estar permeable para asegurar su vitalidad, aunque si se secciona durante la mastectomía o disección axilar aún puede ser usada sobre la base del flujo reverso desde colaterales del serrato mayor ${ }^{6}$.

El colgajo de dorsal ancho es un colgajo muy seguro, se ha convertido en caballo de batalla de la cirugía oncológica reconstructiva en numerosos centros de todo el mundo debido a sus innumerables ventajas en comparación con otras técnicas de reconstrucción, entre las que se menciona la reconstrucción inmediata de grandes defectos, su uso pediculado y libre, disposición de grandes superficies de piel (hasta de $30 \times 20 \mathrm{~cm}$ ), piel desprovista de vellos, sitio donante que no afecta la apariencia del paciente (importante sobre todo en la mujer $)^{3}$, rara vez se describe isquemia o pérdida del colgajo, sus principales limitaciones son su volumen reducido que es capaz de ofrecer y la menor cantidad de piel que puede incluirse como isla en comparación con el colgajo TRAM pero esta paciente es delgada y no tenía tejido abdominal adecuado como para realizar un TRAM.

En el intraoperatorio se realizó la extracción de una barra de estabilización, la que se encontraba expuesta, se talló colgajo de dorsal ancho musculocutáneo con una pastilla de piel voluminosa y tratando de aprovechar todo el músculo para así lograr que el mismo llegue a cubrir la zona esternal (Figuras 4 y 5). La paciente presentó buena evolución en el posoperatorio,

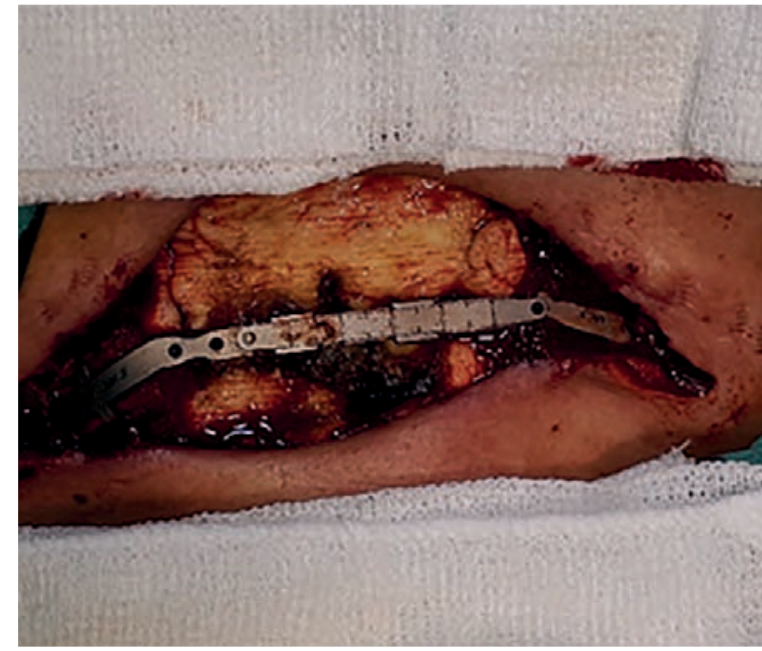

Figura 3. Exposición de material barras estabilizadoras, cemento óseo y malla de doble contacto.

con un colgajo vital, y a pesar de la colocación de los drenajes coleccionó un seroma a nivel de la zona dadora (Figura 6).

Al poco tiempo de reconstruida la pared torácica con el colgajo de dorsal ancho, la paciente presentó dehiscencia de la sutura presternal con desinserción del musculo dorsal ancho y exposición de cemento óseo y de una nueva barra más inferior, La fijación rígida con metacrilato o prótesis metálicas ha sido tradicionalmente considerada como el mejor método para lograr la estabilidad de la pared torácica y prevenir las complicaciones respiratorias. No obstante, si surge una infección, la presencia de material extraño rígido complica significativamente el manejo ${ }^{7}$. En consecuencia, una reparación ideal debería mantener adecuadamente la estabilidad de la pared torácica, para prevenir las complicaciones pulmonares, pero evitando la fijación rígida, motivo por el cual se procedió a retirar todo el material rígido y reinsertar el colgajo ya con el tórax estabilizado, con buena evolución y cobertura del defecto. 


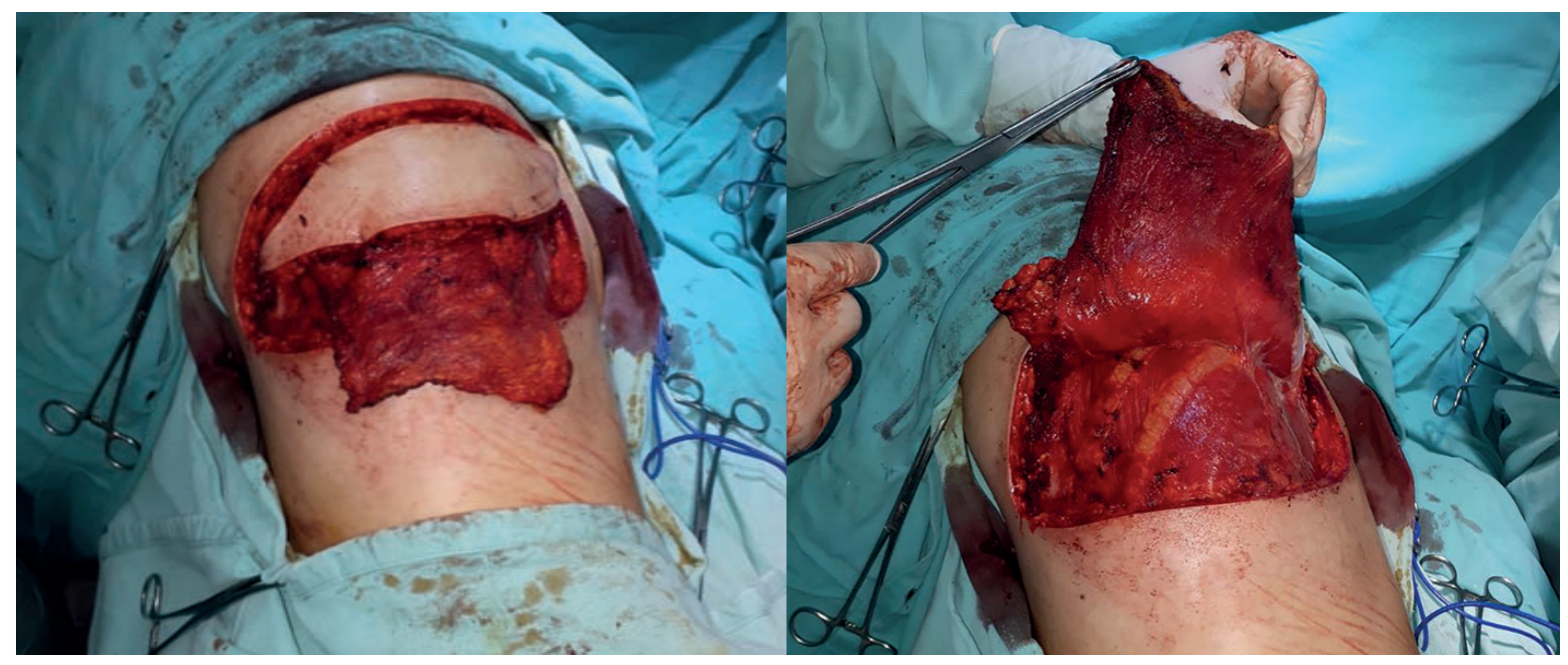

Figuras 4 y 5. Colgajo dorsal ancho con pastilla de piel.

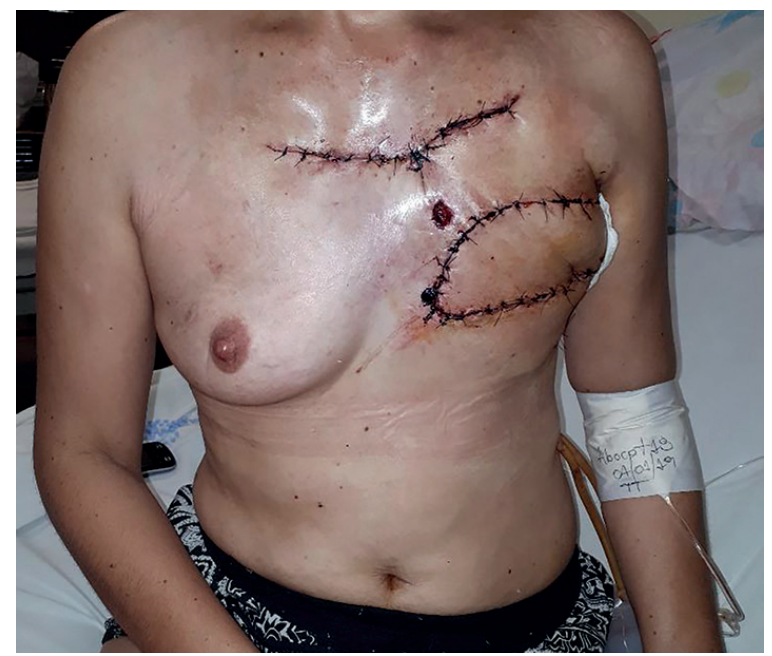

Figura 6. Posoperatorio inmediato, buena vitalidad del colgajo.

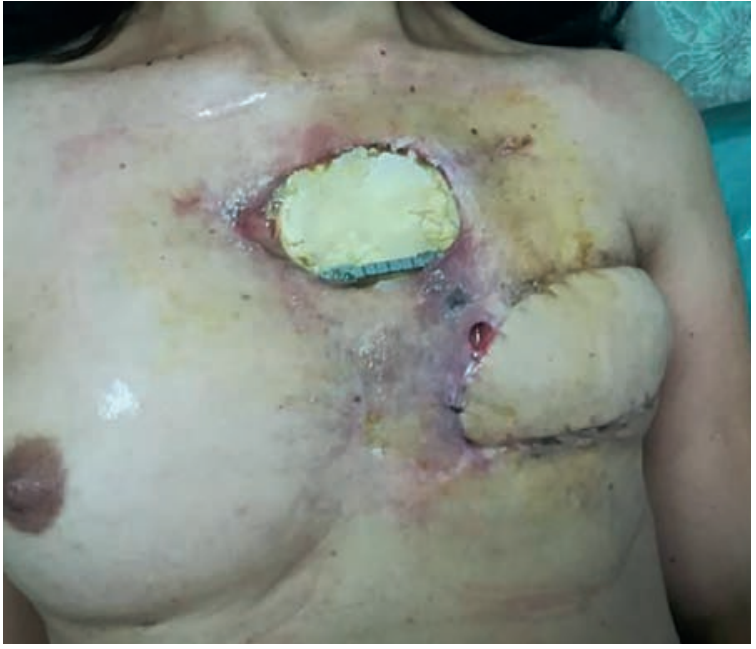

Figura 7. Dehiscencia con exposición de barra y material nuevamente que requiere nueva intervención para retirar el resto del material y reposición del colgajo.

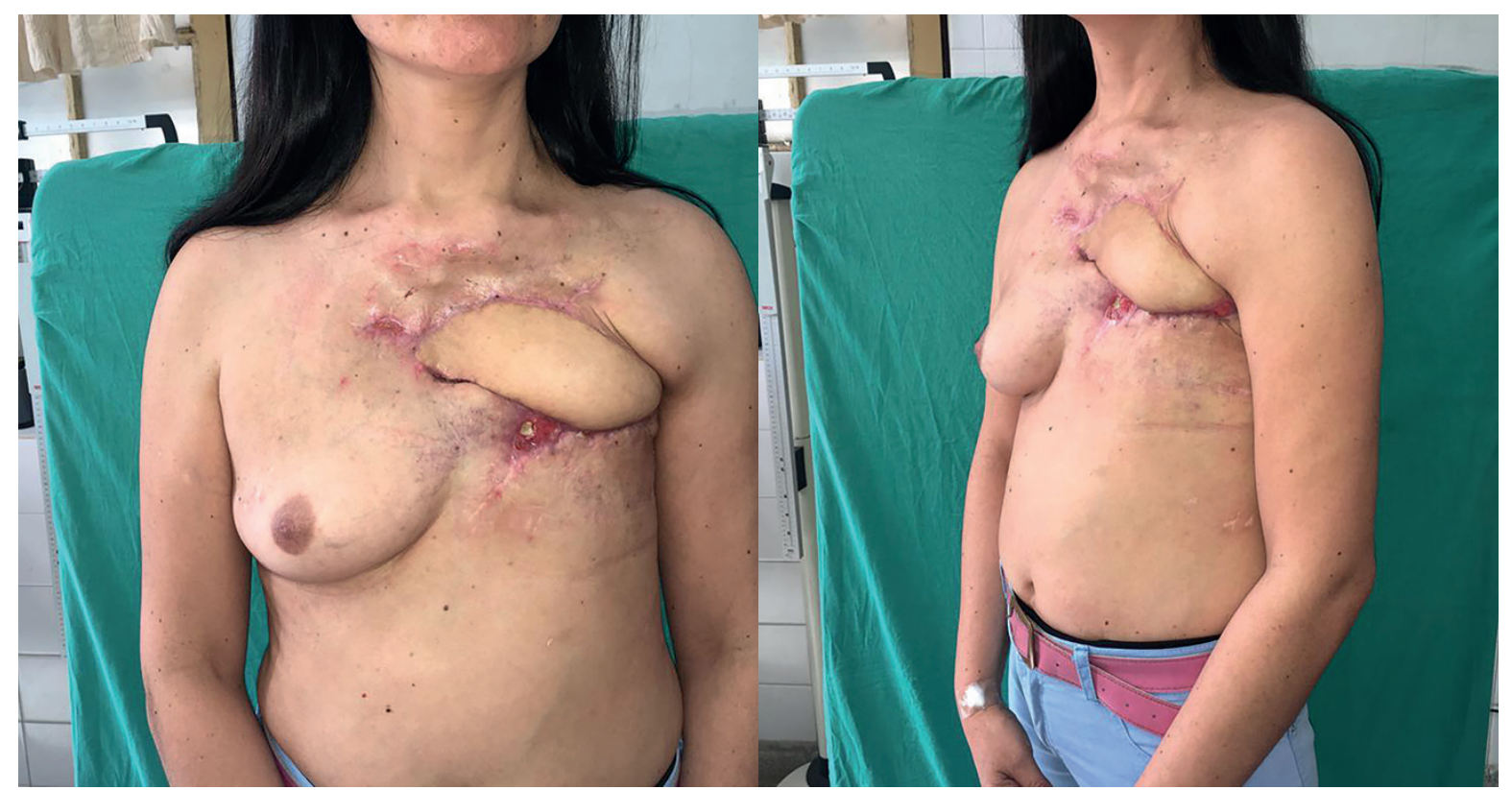

Figuras 8 y 9. Paciente en la actualidad libre de enfermedad con cobertura adecuada de la pared torácica en plan de rehabilitación. 


\section{RESULTADOS}

Actualmente la paciente presenta el colgajo vital con retracción cicatrizal, en su porción inferior presenta una exposición costal que se está manejando con curación oclusiva húmeda en vías de granulación, la paciente recupero peso, no presenta dolor, acude semanalmente a control por el servicio de cirugía plástica.

\section{CONCLUSIONES}

El tratamiento del cáncer de mama es multidisciplinario y puede incluir cirugía, radiación y terapia sistémica (quimioterapia, terapia hormonal o terapia biológica $)^{4}$. En los últimos 30 años, el manejo quirúrgico de la mama ha he- cho una transición desde los abordajes radicales a procedimientos menos extremos, conservadores de la mama, aunque recientemente ha habido una tendencia al aumento de la tasa de mastectomía, por razones no claras.

En este caso en particular se trató de lograr el confort de la paciente y una mejora en su calidad de vida logrando la cobertura del defecto torácico a pesar de la interconsulta tardía ya que se deberían tratar estos casos en conjunto con un equipo multidisciplinario.

Cada caso de cáncer de mama recién diagnosticado debería ser presentado en una conferencia multidisciplinaria para asegurar el manejo óptimo por todas las especialidades involucradas. El tratamiento del cáncer de mama continuará evolucionando; las mejoras adicionales en los resultados se basarán probablemente en el desarrollo de terapias específicas.

\section{BIBLIOGRAFÍA}

1. Cáncer de Mama Dr. Claudio Lorusso Especialista en Mastologia Médico de Staff del Servicio de Ginecología. Hospital Italiano de Buenos Aires Jefe Sección de Patología Mamaria Dra. Karina Pesce Especialista en Mastologia Medica Asociada del Servicio de Ginecología, Sección Patología Mamaria Hospital Italiano de Buenos Aires

2. Colgajo miocutáneo pediculado del dorsal ancho en cirugía de cabeza y cuello Luis Ferbeyre-Binelf

3. Cáncer de mama articulo revisión: diagnóstico, tratamiento y seguimiento de las pacientes con cáncer de mama.Autor: Dr. Matsen CB, Neumayer LA JAMA Surg 2013; 148(10): 971-979
4. Algoritmo en reconstrucción mamaria no inmediata autor: Farjat, Si nijer, Guerrisi, Cirugia Plastica y Reconstructiva vol XXI N25

5. Complicaciones después de mastectomía y reconstrucción mamaria inmediata para el cáncer de mamaAutor: Jagsi $R$, Jiang J, Momoh AO, Alderman A, Giordano SH, Buchholz TA, Pierce LJ, Kronowitz SJ, Smith BD Ann Surg 2016; 263(2): 219-227

6. Cirugía Plástica, Reconstructiva y Estética - Mamas y tronco Profesor. Dr. Felipe Coiffman, FACS

7. Reconstrucción después de una resección mayor de la pared torácicaAutor: Dres. Hanna WC, Ferri LE, MCKendy KM, Turcotte R, Sirois C, Mulder DS Surgery 2011; 150(4): 590-597 